\title{
HIPPOCAMPAL DYSFUNCTION IN SYSTEMIC SCLEROSIS: A LONGITUDINAL PROTON MAGNETIC RESONANCE SPECTROSCOPIC IMAGING ('H-MRSI) STUDY
}

Danilo Rodrigues Pereira ${ }^{1}$, Renan Bazuco Frittoli ${ }^{1}$, Mariana Freschi ${ }^{1}$, Tiago Amaral ${ }^{1}$, Sergio Dertkigil ${ }^{1}$, Ana Paula del Rio ${ }^{1}$, Paula Fernandes Teixeira ${ }^{1}$, Leticia Rittner ${ }^{1}$, Simone Appenzeller ${ }^{1, \star}$

1.Universidade Estadual de Campinas, Campinas (SP), Brazil.

*Corresponding author: appenzellersimone@gmail.com

\section{BACKGROUND}

Systemic sclerosis (SSc) is a multisystem disease characterized by functional and structural abnormalities of small blood vessels, fibrosis of the skin and internal organs. Proton magnetic resonance spectroscopic imaging ( $\left.{ }^{1} \mathrm{H}-\mathrm{MRSI}\right)$ is an important non-invasive method of quantification of biological metabolites. The purpose of this study was to investigate the presence of hippocampal dysfunction in SSc patients using ${ }^{1} \mathrm{H}-\mathrm{MRSI}$ and to determine clinical, laboratory and treatment features associated with its occurrences.

\section{MATERIALS AND METHODS}

We included 36 SSc patients (mean age of 53.46) from our rheumatology outpatient unit and 36 controls (mean age of 48.41). We performed ${ }^{1} \mathrm{H}-\mathrm{MRSI}$ over the bilateral hippocampus and signals from $\mathrm{N}$-acetylaspartate (NAA), choline (Cho), creatine (Cr), glutamate (Glu), glutamine (GIn) and Glx (the sum of Glu and GIn) were measured to determine NAA/Cr, Cho/Cr and GIx/Cr ratios using TARQUIN software. The quality of the spectra was made using metrics: Cramer-Rao lower bound (CRLB), full width half maximum (FWHM), signal-to-noise ratios (SNR) and spectra with low spectral quality were excluded from the analysis (CRLB $>20$, FWHM $>8$ and SNR <10). A complete clinical, laboratory and treatment was performed in all patients. Neurological manifestations were analyzed according to the ACR classification criteria, disease activity (Valentini activity index) and severity activity (Medsger severity index). Cognitive evaluation was performed using the Montreal Cognitive Assessment (MoCA), mood disorders were determined through Beck's depression and Beck's anxiety inventories.

\section{RESULTS}

We observed a significant reduction in NAA/Cr (value $=1.006 ; \mathrm{SD}=0.352$ ) ratios in patients when compared to controls (NAA/Cr value $=1.429, \mathrm{SD}=0.298, \mathrm{p}=0.035)$. Reduction in NAA/Cr ratio was associated with cognitive impairment $(p=0.0170)$, symptoms of anxiety $(p=0.011)$, presence of migraine $(p<0.001)$ and current use of methotrexate $(p=0.028)$. After 48 months, ${ }^{1} \mathrm{H}-\mathrm{MRSI}$ acquisition was repeated in 26 SSc patients at study entry. Follow-up study showed an increase in $\mathrm{Cho/Cr}$ values (Cho/Cr initial: value $=0.242$, Cho/Cr follow-up: value $=0.329, p=0.011$ ) when compared to patient's baseline values

\section{CONCLUSION}

We observed hippocampal dysfunction in SSc. Follow-up study showed changes suggestive of gliosis, indicating irreversible hippocampal dysfunction. 